\title{
QCD Equation of State From a Chiral Hadronic Model Including Quark Degrees of Freedom
}

\author{
Philip Rau ${ }^{* 1,2}$, Jan Steinheimer ${ }^{3}$, Stefan Schramm ${ }^{1}$, Horst Stöcker ${ }^{1,2,4}$ \\ ${ }^{1}$ Frankfurt Institute for Advanced Studies (FIAS), Ruth-Moufang-Str. 1, 60438 Frankfurt am \\ Main, Germany \\ ${ }^{2}$ Institut für Theoretische Physik, Goethe - Universität, Max-von-Laue Str. 1, 60438 Frankfurt \\ am Main, Germany \\ ${ }^{3}$ Lawrence Berkeley National Laboratory, Berkeley, CA 94720, USA \\ ${ }^{4}$ GSI Helmholtzzentrum für Schwerionenforschung GmbH, Planckstr. 1, 64291 Darmstadt, \\ Germany \\ E-mail: raueth.physik.uni-frankfurt.de
}

\begin{abstract}
This work presents an effective model for strongly interacting matter and the QCD equation of state (EoS). The model includes both hadron and quark degrees of freedom and takes into account the transition of chiral symmetry restoration as well as the deconfinement phase transition. At low temperatures $T$ and baryonic densities $\rho_{B}$ a hadron resonance gas is described using a SU(3)flavor sigma-omega model and a quark phase is introduced in analogy to PNJL models for higher $T$ and $\rho_{B}$. In this way, the correct asymptotic degrees of freedom are used in a wide range of $T$ and $\rho_{B}$. Here, results of this model concerning the chiral and deconfinement phase transitions and thermodynamic model properties are presented. Large hadron resonance multiplicities in the transition region emphasize the importance of heavy-mass resonance states in this region and their impact on the chiral transition behavior. The resulting phase diagram of QCD matter at small chemical potentials is in line with latest lattice QCD and thermal model results.
\end{abstract}

8th International Workshop on Critical Point and Onset of Deconfinement

March 11-15

Napa, California, USA

*Speaker. 


\section{Introduction}

Detailed knowledge on the phase diagram of strongly interacting matter is still scarce and the study of QCD matter, in particular at finite temperature $T$ and baryon density $\rho_{B}$ or chemical potential $\mu_{B}$ attains increasing interest. In addition to the well known ground-state properties, from heavy-ion experiments with highest collision energies, nuclear matter is known to exhibit properties of a nearly perfect fluid at very high $T$. At $\mu_{B}=0$, lattice QCD predicts a smooth cross-over transition for the restoration of chiral symmetry at $T \approx 160 \mathrm{MeV}$. For all $\mu_{B}>0$, lattice QCD suffers from the fermion sign-problem which hinders to find solutions in this region. To obtain information on QCD matter at finite potentials, expansion methods can be used. However, until today neither from experiment nor from theory there is a clear picture whether the transition possibly changes to first order and a critical end point exists. Therefore, the phase structure of QCD matter under extreme conditions remains object of an ongoing and lively scientific debate.

This work presents results on the QCD phase diagram from an effective chiral flavor SU(3) model and contrasts them to recent results from the lattice. The model includes all known hadronic degrees of freedom as well as a quark-gluon phase implemented in a PNJL-like approach.

\section{Chiral Effective Model}

The $S U$ (3)-flavor chiral effective model [1] unifies a $\sigma$ - $\omega$-model for the hadronic phase using a non-linear realization of chiral symmetry and a PNJL-like approach for the quark phase. The model is based on a mean field Lagrangian $\mathcal{L}=\mathcal{L}_{\text {kin }}+\mathcal{L}_{\text {int }}+\mathcal{L}_{\text {mes }}$ which includes the particles' kinetic energy, the interaction of baryons with scalar $(\sigma, \zeta)$ and vector $(\omega, \phi)$ meson fields $\mathcal{L}_{\text {int }}=$ $-\sum_{i} \bar{\psi}_{i}\left[\gamma_{0}\left(g_{i \omega} \omega^{0}+g_{i \phi} \phi^{0}\right)+m_{i}^{*}\right] \psi_{i}$. Index $i$ runs over the quark flavors $u, d, s$, and all known baryons (octet, decuplet, and heavy-mass baryon resonances with $m \leq 2.6 \mathrm{GeV}$ ). The effective particle masses $m_{i}^{*}=g_{i \sigma} \sigma+g_{i \zeta} \zeta+\delta m_{i}$ and their effective chemical potentials $\mu_{i}^{*}=\mu_{i}-g_{i \omega} \omega-$ $g_{i \phi} \phi$ are generated dynamically (except for small explicit masses $\delta m_{i}$ ) by the coupling to the scalar and the vector fields respectively. With increasing $T$ and $\rho_{B}, \sigma$ decreases, the effective masses decline, and chiral symmetry is restored. The couplings of the baryon octet are fixed such as to reproduce well-known vacuum masses and nuclear saturation properties. All quark couplings are chosen according to the additive quark model and such as to restrain free quarks from the ground state. The baryon resonance couplings (including the decuplet) are scaled by the coefficients $r_{s}, r_{v}$ to the respective couplings of the nucleons via $g_{B \sigma, \zeta}=r_{s} \cdot g_{N \sigma, \zeta}$ and $g_{B \omega, \phi}=r_{v} \cdot g_{N \omega, \phi}$. To obtain a smooth cross-over at $\mu=0$, the scalar coupling is chosen $r_{s} \approx 1$. The resonance vector coupling $r_{v}$ has a large impact on the transition behavior and on the resulting phase diagram. For reasonable values $r_{v} \approx 1$, the chiral transition is a smooth cross-over in the whole $T-\mu$ plane.

The last term of the model Lagrangian includes the vector and scalar meson self interactions as well as explicit chiral symmetry breaking $\mathcal{L}_{\text {mes }}=\mathcal{L}_{\text {vec }}+\mathcal{L}_{0}+\mathcal{L}_{\text {ESB }}$. All thermodynamic quantities are derived from the grand canonical potential $\Omega / V=-\mathcal{L}_{\text {int }}-\mathcal{L}_{\text {mes }}+\Omega_{\mathrm{th}} / V-U_{\mathrm{Pol}}$, including the thermal contribution $\Omega_{\text {th }}$ of hadrons and quarks which couple to the Polyakov loop $\Phi$. Furthermore, $\Omega$ includes the Polyakov loop potential $U_{\mathrm{Pol}}$ defining the quark dynamics in the model [2]. The shift of degrees of freedom from a hadron resonance gas (HRG) to a pure gas of quarks and gluons is realized by excluded volume effects assuming finite-volume hadrons and point-like quarks. Due to 


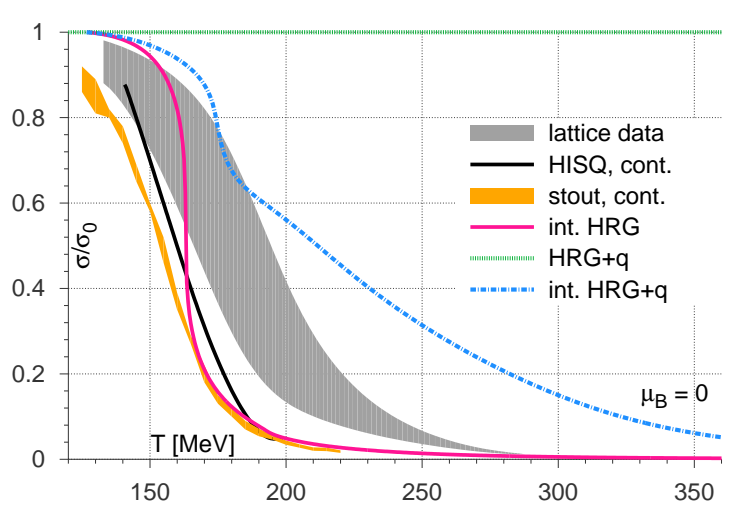

(I)

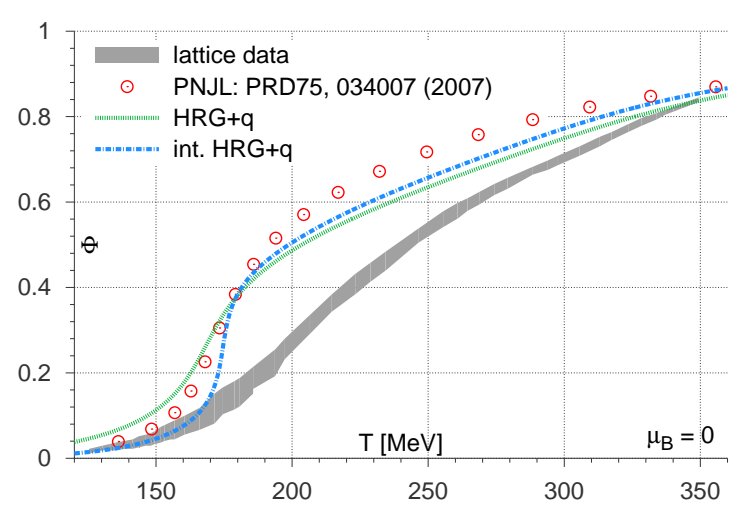

(II)

Figure 1: Normalized chiral condensate (I) compared to older (gray band) and newest continuum extrapolated lattice data [3]. The Polyakov loop from the effective model (analogously to PNJL models [2]) shows a more rapid shift to deconfined quarks as in lattice QCD (II).

the excluded volume, at high $T$ and $\mu$ hadrons vanish from the system and the QGP-phase prevails. Introducing volume correction factors ensures thermodynamics consistency.

\section{Results}

Fig. 1 (I) shows the chiral order parameter normalized by its ground state value $\sigma / \sigma_{0}$ as a function of $T$. The Polyakov loop order parameter $\Phi$ is shown in Fig. 1 (II). The chiral model results are compared to lattice QCD with different fermion actions [3]. The illustration contrasts different parameter sets and particle compositions of the chiral model. A pure interacting HRG without quarks (int. HRG) exhibits the steepest (and continuous) incline of $\sigma$ within a small temperature range. In line with most recent lattice results, a critical temperature $T_{c}=164 \mathrm{MeV}$ is determined.

Other model scenarios additionally include the PNJL-like quark phase with either no interactions between meson fields and particles $(\mathrm{HRG}+\mathrm{q})$ or full interactions (int. $\mathrm{HRG}+\mathrm{q})$. While in the non-interacting case $\sigma$ stays at its ground state value, the decrease of $\sigma$ in the int. HRG+q scenario is rather steep in the beginning. However, as soon as quarks are abundant above the critical Polyakov temperature $T_{0}=175 \mathrm{MeV}$ (Fig. 1 (II)), the slope of $\sigma(T)$ flattens significantly. Comparing the full model results to pure HRG, apparently, hadrons define the slope of $\sigma(T)$ at least up to $T_{c}$ and, thus, the chiral transition is driven by hadrons to a large extent. Due to quarks lacking an eigenvolume, they are preferentially populated at $T \geq T_{c}$ and the number of quarks exceeds the number of hadrons (Fig. 2 (a)). In this region, the significantly smaller quark scalar coupling causes $\sigma$ to decrease much slower. However, as illustrated in Fig. 1 (II), the rapid increase of quark multiplicities at $T_{c}$ generates a distinctly faster increase of $\Phi$ than predicted by lattice QCD (compiled in the gray band). A fundamental discrepancy between $\Phi$ from PNJL models [2] and lattice QCD [3] in the transition region is apparent.

Quantifying the impact of heavy-mass resonances at the phase transition, Fig. 2 (I) shows the baryon number density of baryons (a) and mesons (b) together with quarks divided by $T^{3}$ at $\mu=0$. The total densities (gray line) are broken down into contributions from low- and intermediate-mass particles as well as heavy-mass resonances and the red line illustrates the total quark density. 


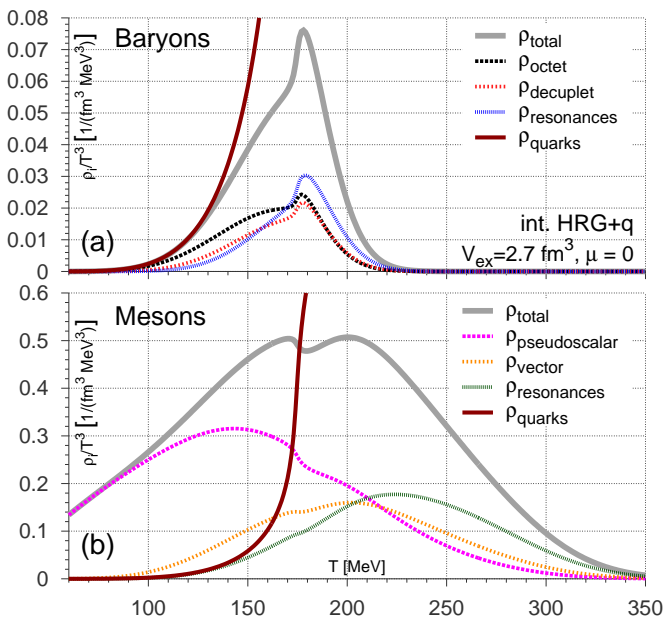

(I)

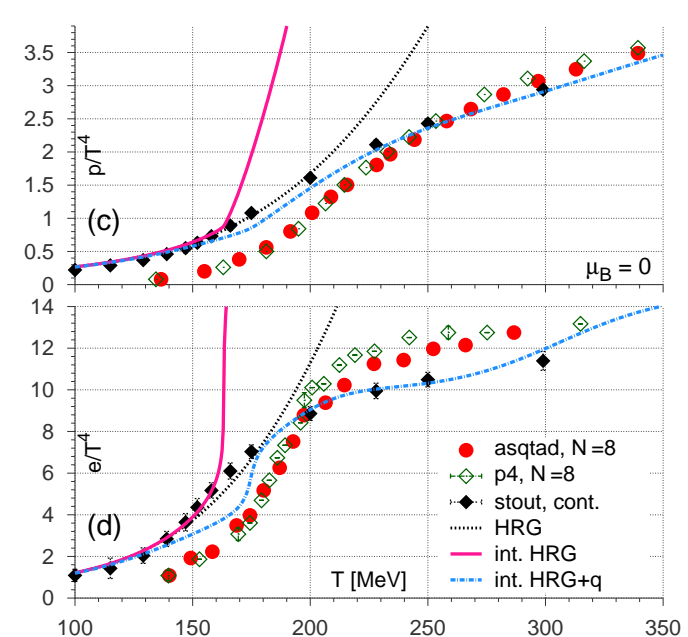

(II)

Figure 2: Total baryon number density of baryons (a), mesons (b), and quarks divided by $T^{3}$ at $\mu=0$. Illustrated are the total densities (gray lines) and the density shares of different particle species. Panels (c) and (d) depict pressure and energy density over $T^{4}$ from different model scenarios contrasted to lattice QCD as functions of $T$.

Below $T_{c}$, low-mass baryons from the octet, mainly nucleons, represent the largest share of the total baryon density (Fig. 2 (a)). This changes as soon as $m^{*}$ of the resonances decline due to a decreasing $\sigma$-field. At $T \geq T_{c}$, heavy-mass resonances are the most abundant baryons in the system. Even though $\Delta$-resonances reach rather large multiplicities with increasing temperature, the combined density contribution of the decuplet stays lowest at all $T$. Regarding the mesons (Fig. 2 (b)), due to the abundant light-mass pions below $T_{c}$, pseudoscalar meson multiplicities are dominant in this region. At $T_{c}$, the fast rising quark number and the pseudoscalar meson mass scaling $m_{\text {mes }}^{* 2} \sim$ $1 / \sigma$ cause pseudoscalar meson multiplicities to decrease as well as a perceivable dip in the total meson density. Higher temperatures are accompanied by an increase in the vector meson densities and a distinctive appearance of heavier meson resonances which become the most abundant mesons at $T \approx 210 \mathrm{MeV}$. This finding of large resonance multiplicities at $T_{c}$ and slightly above underlines the substantial impact of heavy-mass resonances on the chiral transition. The effect of resonances is substantial even in the presence of quarks, where excluded volume effects suppress hadrons at high $T$, and should be even larger in the pure HRG. Due to their large influence, heavy-mass hadron resonances must be considered when studying the phase transition region. Notwithstanding this study only considers $\mu=0$, this conclusion should also hold true in the whole $T-\mu_{B}$-plane.

Fig. 2 (II) shows the pressure $p$ (c) and the energy density $e$ (d) divided by $T^{4}$. For both quantities the interacting pure HRG perfectly reproduces continuum extrapolated lattice QCD results up to $T_{c}$. Also the non-interacting HRG yields a qualitatively good agreement up to slightly higher temperatures. In the whole temperature range, the fully interacting model including quarks yields reasonable results with only minor deviations from lattice in $e / T^{4}$ around $T_{c}$. This dip in $e / T^{4}$ at $T=(155 \pm 15) \mathrm{MeV}$ is caused by the hard-core repulsion of the hadrons. The quark density is just starting to rise sharply and can not compensate the lower hadron contribution in this region. Therefore, the energy density flattens up to $T_{c}$ and rises sharply again thereafter. 


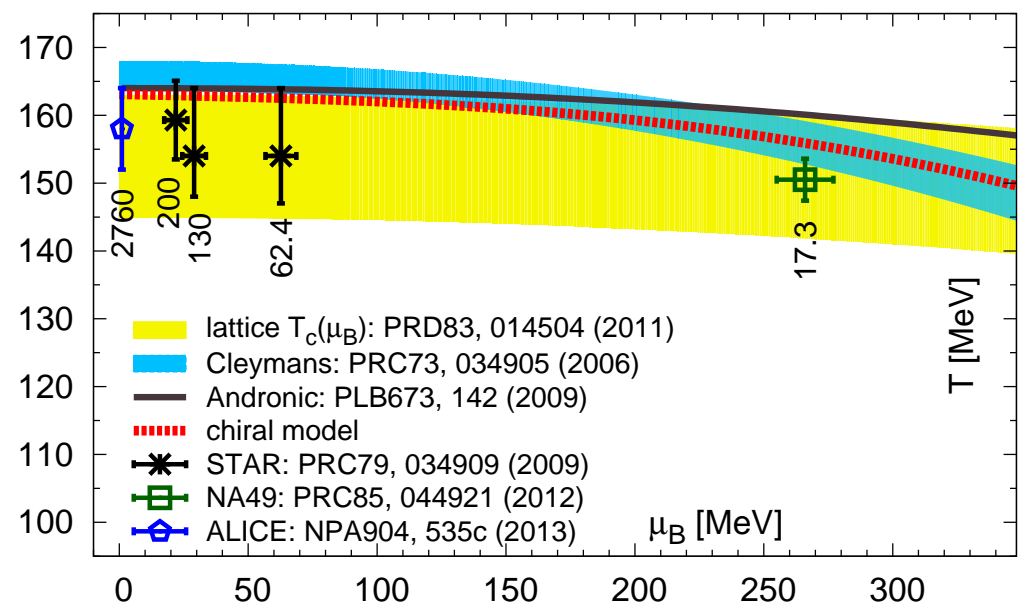

Figure 3: Chiral transition from lattice QCD [4] (yellow band) and from the chiral effective model (red line) at small $\mu_{B}$. The estimates for the transition are compared to freeze-out curves from statistical models and to data from thermal model fits [5] for SPS to LHC energies $\left(\sqrt{s_{\mathrm{NN}}}\right.$ in GeV).

Due to the large number of hadron degrees of freedom, the chiral transition is a smooth crossover even at large $\mu_{B}$. In comparison to lattice QCD extrapolations [4] and thermal model fits of experimental results [5], the phase transition line from the chiral model (Fig. 3) yields a plausible estimate located at the upper boundary of lattice QCD results.

In summary, this work presented a unified effective model which not only provides the correct ground state properties but also describes the transition from a confined HRG to a deconfined quarkgluon phase reasonably well and in line with latest lattice QCD results. An extracted hadron-quark EoS is to be used within dynamic models to study nuclear matter properties in heavy-ion collisions.

\section{References}

[1] J. Boguta and H. Stöcker, Phys. Lett. B120, 289 (1983); P. Papazoglou et al., Phys. Rev. C57, 2576 (1998); Phys. Rev. C59, 411 (1999); J. Steinheimer et al., arXiv:0909.4421; P. Rau et al., arXiv:1302.3836.

[2] C. Ratti et al., Eur. Phys. J. C49, 213 (2007); S. Roessner et al., Phys. Rev. D75, 034007 (2007).

[3] A. Bazavov et al., Phys. Rev. D80, 014504 (2009); Y. Aoki et al. JHEP 0906, 088 (2009); M. Cheng et al., Phys. Rev. D81, 054504 (2010); S. Borsanyi et al., JHEP 1011, 077 (2010); A. Bazavov et al., Phys. Rev. D85, 054503 (2012).

[4] O. Kaczmarek et al., Phys. Rev. D83, 014504 (2011); A. Bazavov et al., Phys. Rev. D85, 054503 (2012).

[5] J. Cleymans et al., Phys. Rev. C73, 034905 (2006); D. Zschiesche et al., J. Phys. G34, 1665 (2007); A. Andronic et al., Phys. Lett. B673, 142 (2009); B. I. Abelev et al. [STAR], Phys. Rev. C79, 034909 (2009); F. Becattini et al., Phys. Rev. C85, 044921 (2012); A. Andronic et al., Nucl. Phys. A904-905, 535c (2013). 